\title{
Structural morphology of amorphous conducting carbon film
}

\author{
P N VISHWAKARMA, V PRASAD, S V SUBRAMANYAM* and V GANESAN ${ }^{\dagger}$ \\ Department of Physics, Indian Institute of Science, Bangalore 560 012, India \\ ${ }^{\dagger}$ Inter University Consortium for DAE Facilities, Indore 452 017, India
}

MS received 17 June 2005

\begin{abstract}
Amorphous conducting carbon films deposited over quartz substrates were analysed using X-ray diffraction and AFM technique. X-ray diffraction data reveal disorder and roughness in the plane of graphene sheet as compared to that of graphite. This roughness increases with decrease in preparation temperature. The AFM data shows surface roughness of carbon films depending on preparation temperatures. The surface roughness increases with decrease in preparation temperature. Also some nucleating islands were seen on the samples prepared at $900^{\circ} \mathrm{C}$, which are not present on the films prepared at $700^{\circ} \mathrm{C}$. Detailed analysis of these islands reveals distorted graphitic lattice arrangement. So we believe these islands to be nucleating graphitic. Power spectrum density (PSD) analysis of the carbon surface indicates a transition from the nonlinear growth mode to linear surface-diffusion dominated growth mode resulting in a relatively smoother surface as one moves from low preparation temperature to high preparation temperature. The amorphous carbon films deposited over a rough quartz substrate reveal nucleating diamond like structures. The density of these nucleating diamond like structures was found to be independent of substrate temperature $\left(700-900^{\circ} \mathrm{C}\right)$.
\end{abstract}

Keywords. Carbon; amorphous; AFM; FFT; X-ray diffraction.

\section{Introduction}

Besides the crystalline forms, amorphous form of carbon has been found to be having a lot of technological applications. The properties of these amorphous carbons sensitively depend on the relative concentration of $s p 3$ and $s p 2$ hybridized carbons. The resulting amorphous materials are variously referred to as tetrahedral amorphous carbon ( $t a-\mathrm{C})$, amorphous carbon $(a-\mathrm{C})$, hydrogenated amorphous carbon $(a-\mathrm{C}: \mathrm{H})$, amorphous conducting carbon $(a-\mathrm{CC})$, etc. In general the carbon films rich in $s p 3$ carbon atoms are referred to as diamond like carbons (DLC). Amorphous carbon with $s p 2$ bonding is always present together with fullerenes, nanotubes and $s p 3$ carbon rich forms, in almost every way of preparation. Because of vast interest in nanotubes and sp3 rich amorphous carbons for their application in field emission, device application, etc investigations on $s p 2$ rich amorphous carbon forms are very few. Though DLC films have potential application in field emission (FE) due to their low threshold voltage, the carbon centres, which are believed to play an important role in controlling and enhancing FE properties of diamond films, are sp2 in nature (Merkulov et al 1999; Obraztsov et al 2000). It is also believed that actual source of emission sites in DLC films are crystalline graphitic phase with $s p 2$ bonding which is mainly present in the grain boundaries and $s p 3$ carbon centres just provide very

*Author for correspondence (svs@ physics.iisc.ernet.in) good medium for heat dissipation due to their high thermal conductivity (Umehara et al 2002). So based on these observations, one can think of a better alternative material for field emission application consisting mainly of $s p 2$ carbon atoms and only little $s p 3$ carbon atoms just enough to dissipate heat produced during field emission. The amorphous conducting carbon films are very much different from DLC films and have predominant network of $s p 2$ carbon atoms whereas in the DLC it is $s p 3$ carbon atoms. Also the hydrogen content in these carbon films was found to be only $1 \%$ (C900) to $2 \%$ (C700) whereas it goes up to $40 \%$ in DLC films. So the name amorphous conducting carbon $(a-C C)$ has been given to distinguish it from other forms of amorphous carbon mainly consisting of sp3 carbon atoms (Subramanyam 1996).

Miniaturization of electronic device fabrication has been possible using nanotubes and self assembled molecules (Bachtold et al 2001; Javey et al 2004). For devices out of nanotubes, the electrodes to the logic gates and to the devices are made with thin films of $\mathrm{Au}, \mathrm{Pt}$, etc. However, due to mismatch in Fermi levels of the device material and the electrode, it is always advisable to have electrodes of same material as that of the device. Metallic nanotubes can also serve this purpose. However, its one dimensionality and cost of production for pure and defect free metallic nanotubes limits its application as electrodes for nano-electronic circuits. Here $a$-CC seems to be a perfect candidate for such purposes owing to its low cost of production and high conducting nature. Though one can choose various methods to prepare $a$-CC, we 
have adopted pyrolysis assisted CVD method due to its simplicity and low-cost and high yield of $a$-CC. Since the properties of these conducting carbon films are very much different from DLC films and hence the surface of these films is also expected to be different. The conductivity of $a$-CC varies from $\sigma=10 \mathrm{~S} / \mathrm{cm}$ to $300 \mathrm{~S} / \mathrm{cm} \mathrm{de-}$ pending upon the preparation temperature. It was found that disorder-induced localization of electrons in amorphous conducting carbon films was responsible for MI transition in them (Sayeed 1998). So by varying the preparation conditions, the physical and chemical properties of $a$-CC films can be tuned for the desired technological applications. Because many physical and chemical properties such as electrical, optical are sensitive to the surface morphology, detailed knowledge and precise control over the surface roughness is of great importance. So in this paper we are presenting a comparative study of the structural changes including the surface of $a$-CC prepared under various conditions.

\section{Experimental}

Amorphous conducting carbon films were prepared by using high temperature pyrolysis assisted CVD of maleic anhydride $\left(\mathrm{C}_{4} \mathrm{H}_{2} \mathrm{O}_{3}\right)$ in argon atmosphere using two-zone furnace arrangement. About $100 \mathrm{mg}$ of maleic anhydride was taken in a $50 \times 1 \mathrm{~cm}$ quartz tube closed at one end. Polished quartz substrate $(5 \times 5 \mathrm{~mm})$, ultrasonically cleaned in water followed by acetone was positioned at the centre of first zone of furnace inside the quartz tube. The open end of the tube was closed with a rubber bladder. The quartz tube was evacuated and purged with argon gas prior to pyrolysis. The pyrolysis process was performed for $5 \mathrm{~h}$ and then the furnace was switched off and allowed

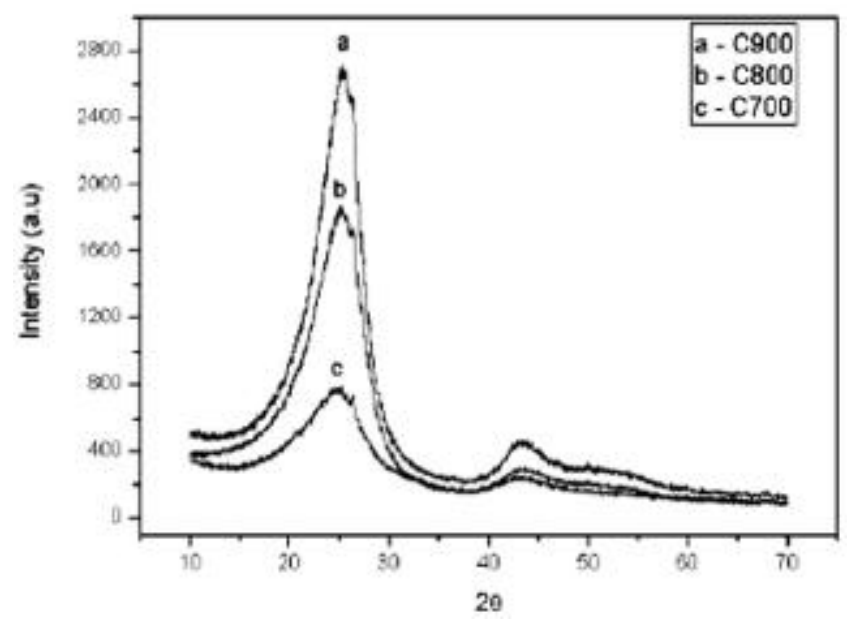

Figure 1. X-ray diffraction plot for C700, C800 and C900 ( $y$ axis is relatively displaced for clarity). Shift in peak near $2 \theta=25^{\circ}$ towards higher angle for samples prepared at higher temperature shows flatness in the layers of graphine sheet. to cool under normal ambient conditions. The residual gas consisting of mainly $\mathrm{CO}$ and $\mathrm{CO}_{2}$ were collected in the rubber bladder. This process was repeated for three different pyrolysis temperatures viz. $700^{\circ} \mathrm{C}(\mathrm{C} 700), 800^{\circ} \mathrm{C}$ (C800) and $900^{\circ} \mathrm{C}(\mathrm{C} 900)$. The thickness of the films deposited over the smooth quartz substrate was $\sim 1 \mu \mathrm{m}$. Surface analysis of these films was carried out using AFM Nanoscope-E, Digital Instruments, with 10-micron scanner-high resolution. Cantilever used was about $115 \mathrm{mi}-$ cron from the substrate-apex of the triangular section and is of silicon nitride $\left(\mathrm{Si}_{3} \mathrm{~N}_{4}\right)$, same as the tip material. The base of the tip was about $4 \mu$ in radius and about $20 \mathrm{~nm}$ radius at the vertex. The force constant for the entire experiment was about $0.58 \mathrm{~N} / \mathrm{m}$. The force used typically varied from $2-10 \mathrm{nN}$. The area of the scanned sample was $1 \times 1 \mathrm{~mm}$. All the AFM surface scannings were done in contact mode.

\section{Results and discussion}

Besides surface analysis, the samples were characterized using X-ray diffraction for structural changes. Figure 1 shows the X-ray diffraction plot for the samples C700, C800 and C900. XRD patterns of all the samples exhibit mainly two types of peaks: (002) resulting from stacks of parallel layer planes at $25-27^{\circ}$ and two dimensional (10) peaks resulting from the regular structure within the individual layer plane segments at $41-47^{\circ}$. Peaks of the type ( $h k l)$ are absent, indicating that there is little or no stacking order in the arrangement of parallel layers. These patterns are known to be characteristic of disordered graphite like structure of carbon where the graphene layers are regularly stacked in itself but has no correlation to the next pile except for parallelism (Franklin 1950; Ruland 1968). The interlayer spacing increases as one goes from C900 to C700 (table 1). The results have been compared with the standard data of graphite. The simple van der Waals force between the two layers in graphite lattice gives an interlayer spacing of $0.344 \mathrm{~nm}$ and the interaction between the pi-electron clouds of the two layers reduces the interlayer spacing to $0.3354 \mathrm{~nm}$. However, for the above interlayer spacing in graphite, the flatness of the graphite layer planes is essential. Even slightest deviation from a perfect plane can give rise to an interlayer spacing of more than $0.3354 \mathrm{~nm}$. The value of interlayer

Table 1. X-ray diffraction from pyrolytic carbon films compared to those of graphite.

\begin{tabular}{lllc}
\hline Sample & $\begin{array}{c}\text { Peak }(2 \theta) \\
\text { (Degree) }\end{array}$ & $\begin{array}{c}c \\
(\mathrm{~nm})\end{array}$ & $\begin{array}{c}\text { Interlayer spacing } \\
(\mathrm{nm})\end{array}$ \\
\hline C700 & 24.27 & 0.7180 & 0.359 \\
C800 & 24.77 & 0.7179 & 0.359 \\
C900 & 25 & 0.712 & 0.356 \\
Graphite & 26.5 & 0.671 & 0.335 \\
\hline
\end{tabular}


spacing in our system is far from that of graphite suggesting a highly rough graphene plane and the roughness increases as one moves from C900 to C700.

Raman data on these films (Subramanyam et al 1997) suggest that the carbon films formed at $700^{\circ} \mathrm{C}$ are somewhat independently clustered and are slightly graphitic among themselves. When the preparation temperature is increased to $800^{\circ} \mathrm{C}$, these independent clusters start joining together giving Raman spectra similar to that of some polymers. At $900^{\circ} \mathrm{C}$ these clusters coalesce together to form a continuous film.

The 3D surface morphology of C700, C800 and C900 are shown in figure 2. Sample C900 is topologically smoother than that prepared at $\mathrm{C} 800 / \mathrm{C} 700$. Also as we move from $\mathrm{C} 700$ towards $\mathrm{C} 900$ via C800, nucleation of well-defined islands start, which are not very prominent in C700 but are slightly more prominent in C800 and is very clear in C900. A closer look at these nucleating islands gives distorted rhombohedral arrangement of lattice points. However, the long range ordering is not seen in this rhombohedral lattice arrangement.

FFT is a powerful tool for surface roughness analysis. Basically any information whose repetition is more, will lie at higher frequency region of Fourier image whereas any information whose repetition is least or it is hardly repeated will be present at low frequency region of the Fourier image. So for a rough surface, lot of high frequency signals along with low frequency signal is expected whereas for a relatively smooth surface those high frequency signals will be either diminished or absent, and
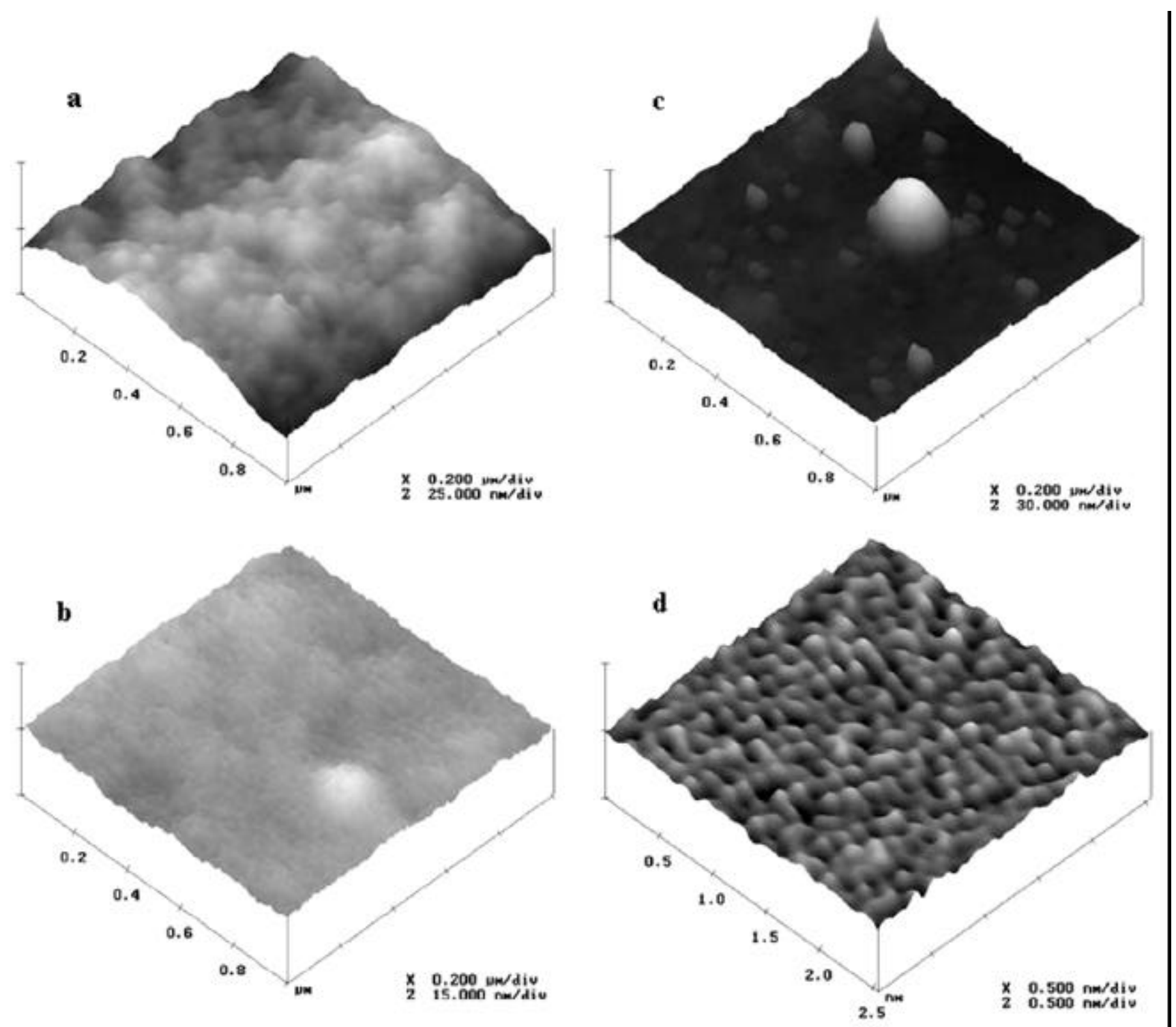

Figure 2. 3D surface morphology of a. C700, b. C800 and c. C900. The surface smoothness decreases from C900 to C800 and to C700. Besides the smooth surface, C900 has some nucleating islands. These islands are not present in C800 and C700. d. Closer look of one of these nucleating islands. 
only low frequency signals will be seen. The central peak, which also represents the d.c. component, will always be present. Figure 3 shows FFT of samples C700, C800 and C900. As can be seen in the figure, a lot of high frequency signals along with low frequency signals are present for C700. As we move from C700 towards C800 and C900, the high frequency signals get diminished. FFT for the nucleating islands gives four peaks besides the central peak which are almost equidistant from each other but does not form a square i.e. its positions can be thought of as at the vertices of a rhombus. Generally one may expect hexagonal arrangement of atoms for graphite. However, rhombohedral structure has been observed on graphite surface (Hembacher et al 2003) and this has been attributed to hidden surface atoms. So we believe that these islands are nucleating graphitic centres, which are prominent only for the samples prepared at high temperature.

The PSD function is defined as the square modulus of the Fourier transform of the surface profile.

$$
\begin{aligned}
& \operatorname{PSD}(u, v)=|F[R(x, y)]|^{2}=F\left[f_{i}(x, y)\right] F\left[f_{j}(x, y)\right]^{*} \\
& \operatorname{PSD}(u, v)=\left|\frac{1}{N_{x} N_{y}} \sum_{x=1}^{N} \sum_{y=1}^{N} f(x, y) e^{2 \pi i\left(\frac{u x}{N_{x}}+\frac{v y}{N_{y}}\right)}\right|^{2} .
\end{aligned}
$$

However, the information is in the frequency domain rather than the spatial domain. For statistically isotropic surfaces which have no scale and whose height is well defined but non-differentiable may indeed have spectra of the fractal form (Berry and Hannay 1978)

$$
\operatorname{PSD}(f)=K_{0} f^{-\gamma},
$$

where $K_{0}$ is a constant (called topethesy (Sayles and Thomas 1978)) dependent on the system and $\gamma$ is related to the scaling exponent, $\alpha$ (also called fractal dimension) by $\alpha=(\gamma-d) / 2$ (Voss 1985), where for the line scan dimension $d=1$. The permissible range of $\gamma$ is 1 to 3 (Feder 1988). Figure 4 shows the 1-D PSD plot for the three samples and linear fitting to the intermediate region giving the value of $\gamma($ or $\alpha)$ for $\mathrm{C} 700=2.07(0.535)$, $\mathrm{C} 800=2.20(0.56)$ and $\mathrm{C} 900=3.35(1 \cdot 175)$. The value of $\gamma$ is related to the smoothness of the surface. Higher the value of $\gamma$, smoother the surface will be. The value of $\alpha$ such that, $0<\alpha<1$, describes a balance between roughening mechanisms, such as random fluctuations in the deposition process and smoothing process, such as diffusion at the growth front so that the local structure remains unchanged (Family and Vicsek 1985; Barabasi and Stanley 1995). The second type is anomalous dynamic scaling, which gives $\alpha>1$ (Wolf and Villain 1990; Amar et al 1993; Lu et al 1995). In this situation the roughening fluctuations and the smoothing effects cannot quite reach a balance and the slope of the surface increases with time. Hence, an increase in the value of $\alpha=0.5$ (C700) to $\alpha=1.2$ (C900) indicates a transition from the nonlinear growth mode to linear surfacediffusion dominated growth mode (Casiraghi et al 2005).

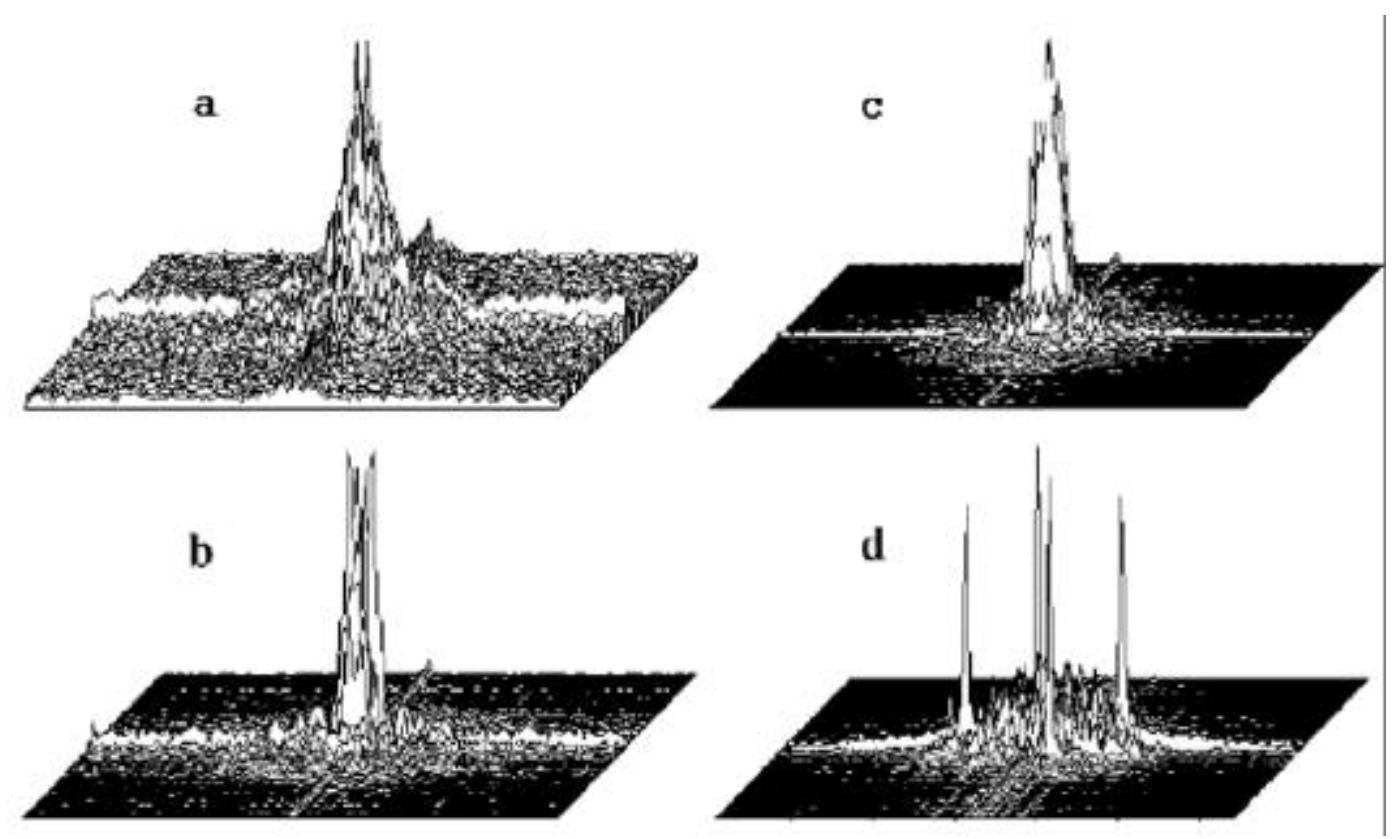

Figure 3. FFT of 2D AFM image of a. C700, b. C800, c. C900 and d. the nucleating islands. FFT of surface of these islands consists of four peaks along with the central peak indicating rhombohedral symmetry of the lattice. 
Figure 5 shows the surface morphology of C700, C800 and C900 films deposited over rough quartz surface. The surface roughness of quartz substrate was about $1 \mu \mathrm{m}$, so the film deposited over the rough substrate was $\sim 10 \mu \mathrm{m}$. The surface morphology of these films is very much different from that on the polished quartz substrates. One thing which is common in the films deposited over pol-
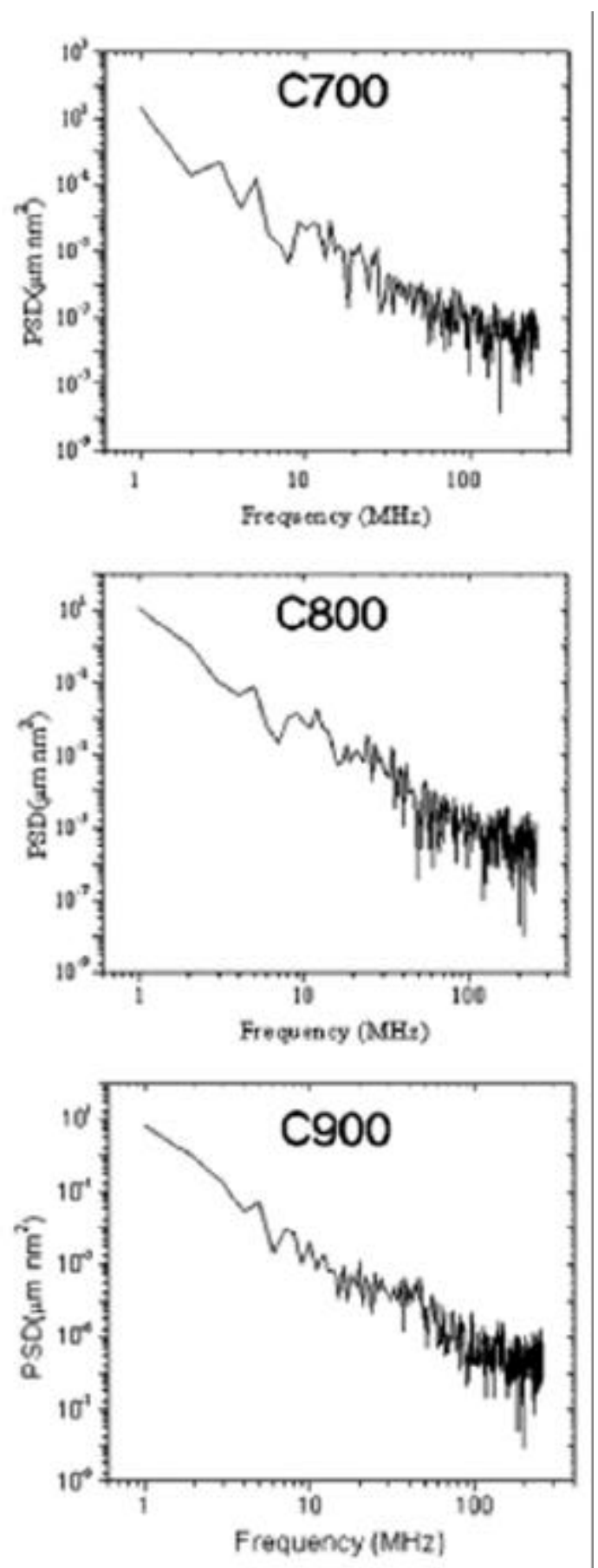

Figure 4. PSD plot for $2 \mathrm{D}-\mathrm{AFM}$ images of C700, C800 and C900. Linear fitting to the intermediate region gives the value of $\alpha 0.535$ (C700), 0.56 (C800) and 1.175 (C900). ished and rough quartz substrates, is that of increasing smoothness of the films as one goes towards higher preparation temperature. In both the cases C900 films are relatively smoother than $\mathrm{C} 700$. Also for the films over rough surface, along with spherical carbon clusters, some sharp edges were also observed. These sharp edges were very clearly seen in C700, C800 and C900. These sharp features are very much similar to that observed for DLC films (Rusop et al 2004). So we believe that these sharp edges formed are in the early stages of diamond formation. The temperature effect on the surface morphology comes only in enhancing the smoothness of the film. The C900 films are relatively smoother than C800 and in turn C700, though the densities of these nucleating diamonds are same for the three samples. The only factor which may be giving rise to diamond nucleation is the predeposition surface roughness. It was observed that irrespective of the growth process stress is an intrinsic property of $a-\mathrm{C}$ films and to minimize the stress some $s p 3$ carbon atoms convert themselves to $s p 2$ configuration (Orwa et al 2004). Other methods of stress reduction employ incorporation of elements such as metals (Dimigen et al 1997), boron (Chhowalla et al 1996), silicon (Racine et al 2001) or post-deposition thermal annealing (Freidmann et al 1997). So the one thing that is common to all the stress reduction methods is introduction of disorder either by foreign element or converting few $s p 3$ carbon atoms to $s p 2$ by thermal means. We believe here also the disorder introduced by surface roughness of the substrates is acting in a similar way to reduce stress and thereby felicitating $s p 3$ carbon atoms to retain their configuration. Whereas on the smooth substrates, there is no such medium available to reduce stress and hence more $s p 2$ configuration forms to minimize stress and making C900 films more graphitic than C800/C700. This is also supported by the a.c. conductivity studies on rough and smooth films. The films on smooth substrate do not show any frequency dependence whereas films over rough substrate show slight frequency dependence at high frequency $(100 \mathrm{kHz})$ (Vishwakarma and Subramanyam, to be published).

\section{Conclusions}

The X-ray diffraction data of the carbon samples show disordered graphite like structure of carbon where the graphene layers are regularly stacked but have no correlation to the next pile except for parallelism. The films prepared at higher temperatures are relatively smoother than those prepared at lower temperature besides nucleating graphitic centres which are not seen in C800 and C700. Power spectrum density (PSD) of the carbon surface indicates a transition from the nonlinear growth mode to linear surface-diffusion dominated growth mode resulting in a relatively smoother surface as 

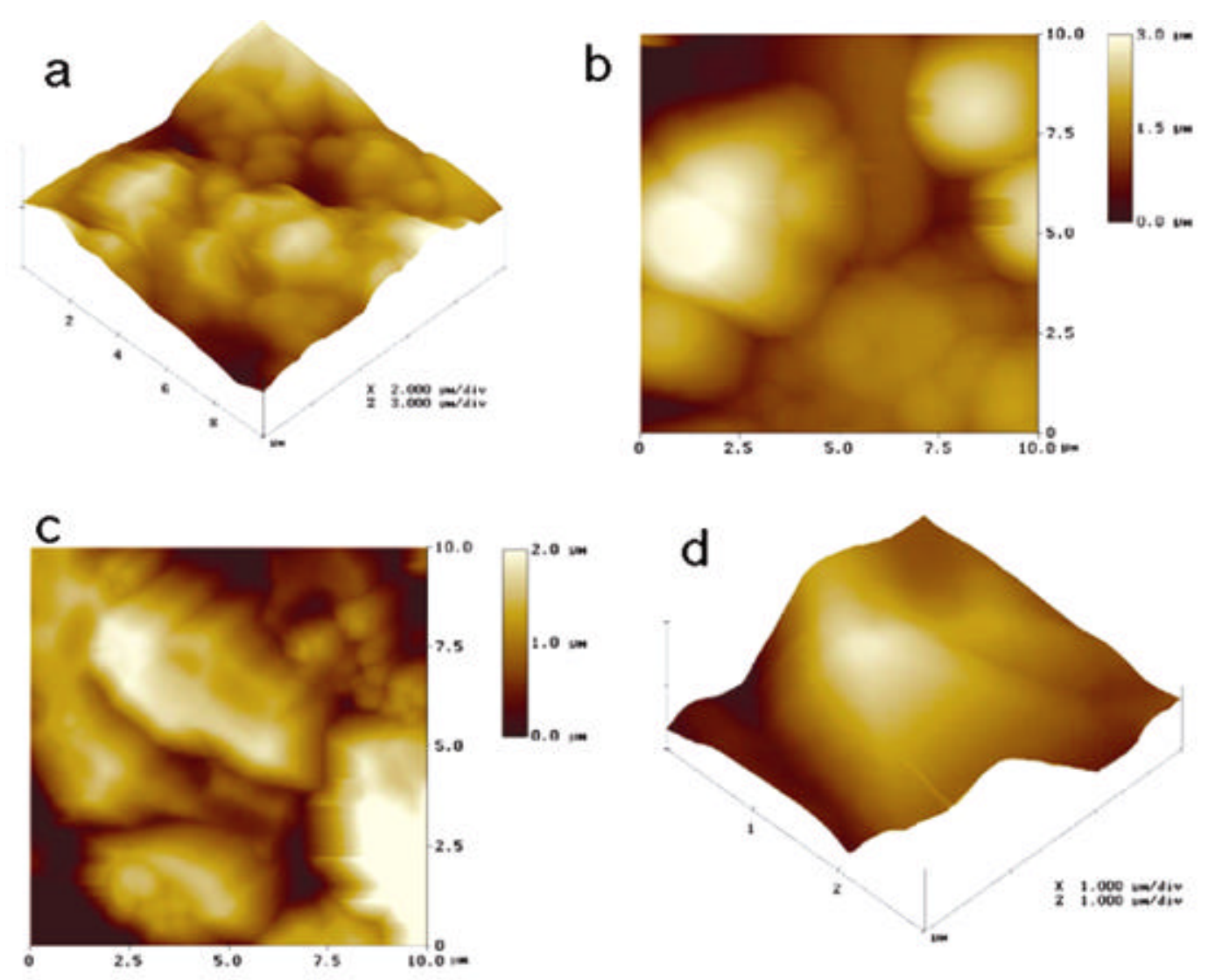

Figure 5. Surface morphology of a. C700, b. C800, c. C900 and d. one nucleating island on rough quartz surface.

one moves from low preparation temperature to high preparation temperature. The surface morphology of $a$ $\mathrm{CC}$ films on rough substrate is different from that on polished substrate, but is very similar to that of nucleating diamond. The number density of this nucleating diamond is same for C700, C800 and C900. So we believe temperature is not playing much role except enhancing the smoothness of the film whereas surface roughness helps in diamond nucleation by reducing strain in the film. An extensive investigation of $a-C$ films on substrate of varying roughness can give a better insight of the role played by surface roughness on the film properties. The frequency dependence of conductivity comes due to more sp3 carbon atoms in films over rough surface than the films on smooth substrate.

\section{References}

Amar J G, Lam P M and Family F 1993 Phys. Rev. E47 3242

Bachtold A, Hadley P, Nakanishi T and Dekker C 2001 Science 2941317

Barabasi A L and Stanley H E 1995 Fractal concepts in surface growth (Cambridge: Cambridge University Press)

Berry M V and Hannay J H 1978 Nature 273573
Casiraghi T C, Ferrari A C and Robertson J 2005 Diamond Relat. Mater. 14913

Chhowalla M, Yin Y, Amaratunga G A J, Mckenzie D R and Frauenheim T 1996 Appl. Phys. Lett. 692344

Dimigen H, Hubsch H and Memming R 1997 Appl. Phys. Lett. 501056

Family F and Vicsek T 1985 J. Phys. A18 L75

Feder J 1988 Fractals (New York: Plenum) Ch. 14

Franklin R E 1950 Acta Crystallogr. 3107

Freidmann T A, Sullivan J P, Knapp J A, Talland D R, Follstaedt D M, Medlin D L and Mirkarimi P B 1997 Appl. Phys. Lett. 713820

Hembacher S, Giessibl F J, Mannhart J and Quate C F 2003 Proc. Nat. Acad. Sci. 10012542

Javey A, Guo J, Farmer D B, Wang Q, Wang D, Gordon R G, Lundstrom M and Dai H 2004 Nanoletters 4447

Lu T M, Yang H N and Wang G C 1995 in Fractal aspects of materials (eds) F Family et al, MRS symposia proceedings No. 367 (Pittsburgh: Materials Research Society) p. 283

Merkulov V I, Lowndes D H and Baylor L R 1999 Appl. Phys. Lett. 751228

Obraztsov A N, Volkov A P and Pavlovsky I 2000 Diamond Relat. Mater. 91190

Orwa J O, Andrienko I, Peng J L, Prawer S, Zhang Y B and Lau S P 2004 J. Appl. Phys. 966286

Racine B, Ferrari A C, Morrison N A, Hutchings I, Milne W I and Robertson J 2001 J. Appl. Phys. 905002 
Ruland W 1968 in Chemistry and physics of carbon (ed.) P L Walker Jr (New York: Marcel Dekker) Vol. 4, pp 184

Rusop M, Mominuzzaman S M, Soga T and Jimbo T 2004 Diamond Relat. Mater. 132180

Sayeed Ahmed 1998 Structural and electrical properties of amorphous conducting carbon films, Ph.D. thesis, Indian Institute of Science, Bangalore

Sayles R S and Thomas T R 1978 Nature 271431
Subramanyam S V 1996 Indian J. Pure \& Appl. Phys. 34 595

Subramanyam S V, Sayeed A, Meenakshi V, Bhattacharya S, Cholli A and Tripathi S 1997 J. Appl. Phys. 812907

Umehara Y, Murai S, Koide Y and Murakami M 2002 Diamond Relat. Mater. 111429

Voss R F 1985 in Scaling phenomena in disordered systems (eds) R Pynn and A Skejeltorp (New York: Plenum) pp 1-11 Wolf D E and Villain J 1990 Europhys. Lett. 13389 